\title{
The role of AIRE polymorphisms in melanoma
}

\author{
G. Conteduca $^{\text {a }}$, F. Ferrera ${ }^{\text {a }}$, L. Pastorino ${ }^{b}$, D. Fenoglio ${ }^{a, c}$, S. Negrini ${ }^{a, c}$, \\ M.P. Sormani ${ }^{\text {d }}$, F. Indiveri ${ }^{a, c}$, G. Bianchi Scarrà ${ }^{b, 1}$, G. Filaci ${ }^{a, c, *, 1}$
}

${ }^{a}$ Center of Excellence for Biomedical Research, University of Genoa, Italy

${ }^{\mathrm{b}}$ Department of Oncology, Biology and Genetics, University of Genoa, Italy

' Department of Internal Medicine, University of Genoa, Italy

d Biostatistics Unit, Department of Health Sciences, University of Genoa, Italy

Received 18 November 2009; accepted with revision 5 March 2010

Available online 2 April 2010

\author{
KEYWORDS \\ AIRE; \\ Melanoma-associated \\ antigens; \\ Medullary thymic \\ epithelial cells; \\ Promiscuously \\ expressed genes
}

\begin{abstract}
Polymorphisms of AIRE, a transcription factor that up-regulates intrathymic expression of tissue-specific antigens including melanoma-associated antigens (MAAs), may variably affect the selection of MAAs-specific thymocytes, generating T-cell repertoires protecting or predisposing individuals to melanoma. We found that AIRE single nucleotide polymorphisms (SNPs) rs1055311, rs1800520 and rs1800522 were significantly more frequent in healthy subjects than in melanoma patients, independently from sex, age and stages of melanoma. The presence of these SNPs was associated with increased frequency of two T-cell clonotypes specific for MAGE-1 linking their protective effect to selection/expansion of MAA-specific T cells. Interestingly, mRNA transcribed on the rs1800520 SNP showed increased free energy than the wild type suggesting that its reduced stability may be responsible for the different activity of the polymorphic AIRE molecule. This finding may contribute at identifying subjects with increased risk of developing melanoma or patients with melanoma that may take benefit from immunotherapy.

(c) 2010 Elsevier Inc. All rights reserved.
\end{abstract}

\section{Introduction}

The AIRE (autoimmune regulator) gene, located in the $21 q 22.3$ region, is approximately $13 \mathrm{~kb}$ in length and contains 14 exons that encode a polypeptide of 545 amino acids [1,2].

AIRE protein acts as a powerful transcriptional transactivator $[3,4]$. In particular, the carboxy-terminal part of AIRE, containing the plant homeodomain (PHD) finger and leucine

\footnotetext{
* Corresponding author. Centre of Excellence for Biomedical Research (CEBR), University of Genoa, Viale Benedetto XV n. 7, 16132 - Genova, Italy. Fax: + 390103533025.

E-mail address: gfilaci@unige.it (G. Filaci).

1 These two authors contributed equally to the manuscript.
}

zipper domain, is supposed to be critical for its transactivation capacity. Other domains of the AIRE protein indicating its involvement in transcriptional processes include the aminoterminal homogeneously staining region (HSR) domain, the nuclear localization signal (NLS) domain, the SAND domain, and four LXXLL motifs $[1,2,5]$. Since in the Sp100 protein family the HSR region is responsible for the dimerization of the polypeptides belonging to these proteins, the HSR domain present in AIRE is suggested to mediate its homo- or multidimerization. The SAND domain is thought to act as a DNA binding domain, and its structure has been determined in a number of nuclear proteins including Sp100 family proteins $[6,7]$. The LXXLL motifs are found in various proteins and are needed for the binding to nuclear receptors, mainly in a ligand- 
dependent manner [8]. Interestingly, the AIRE PHD1 domain has also ubiquitin E3 ligase activity. This finding implies that AIRE might, in addition to its transcriptional regulatory function, also regulate the intracellular proteosome pathway [9].

AIRE mutations cause the rare recessive autoimmune polyendocrinophathy-candidiasis-ectodermal dystrophy syndrome (APECED), also known as autoimmune polyendocrine syndrome-type 1(APS-1). This pathology is a polyglandular disorder that classically manifests as spontaneous autoimmunity against the parathyroid and the adrenal glands associated with mucocutaneous candidiasis infection [10].

Although AIRE is expressed in several tissues, its highest level of expression is in thymus [11-13] where it is seen in a subpopulation of medullary thymic epithelial cells (mTEC) [13]. In mTECs AIRE, binding (directly or indirectly) to specific motifs in promoter-enhancer regions, or inducing chromatin remodeling [14], is responsible for the expression of a large portion of genes known as promiscuously expressed genes. These genes encode for tissue-restricted self-antigens (TRAs), proteins that carry out cell type-specific functions, but which are not necessary for the physiological gene expression program of thymic stromal cells [15]. The expression of TRAs in mTECs allows the negative selection of autoreactive lymphocytes provided with high affinity for the recognized auto-antigen, but not of those with low/ intermediate affinity $[16,17]$. This causes the exportation to the periphery of potentially autoreactive T-cell clones [18-21]. Although this phenomenon can predispose to the onset of autoimmunity, it is fundamental for spontaneous or vaccine-induced generation of anti-cancer immune responses directed against endogenous tumor-associated antigens. Hence, a balanced activity of AIRE is necessary for the immune homeostasis: at variance, hyper-functional molecular forms of AIRE could expose the individual to an overwhelming thymocyte deletion depriving the immune repertoire of clones able to exert irreplaceable effector activity against cancer cells. Indeed, some melanomaassociated antigens (MAA), such as MAGE, Gp100, MART-1, are among TRAs expressed in mTECs [15,22-25]. This suggests that AIRE polymorphisms impacting on their functional activity may condition the thymocyte repertoire selected on the basis of the recognition of intrathymically expressed MAAs.

In this study, we selected the five most frequent AIRE polymorphisms in the European population [rs878081 (C7094T), rs1055311 (C8385T), rs1800520 (C8723G), rs1800525 (G11107A) and rs1800522 (T16366C)] (Fig. 1). Their distribution was analyzed in a series of 70 patients affected with melanoma and in 171 healthy controls in order to determine their potential influence on melanoma susceptibility.

Furthermore, the frequency of two MAGE-1-specific T-cell clonotypes was analyzed in patients carrying less than two AIRE SNPs in comparison with patients carrying two or more AIRE SNPs with aim of getting insights on the possible impact exerted by AIRE polymorphisms on the composition of the MAA-specific T-cell repertoire.

\section{Materials and methods}

\section{Patients and controls}

Seventy patients affected with melanoma ( 35 males and 35 females, age ranging from 15 to 75 years, 34 with stage I-II and 36 with stage III-IV), and 171 healthy control subjects (60 males and 111 females, age ranging from 15 to 75 years) were analyzed in a case-control study. Diagnosis was based on typical clinical findings and histological analysis. Peripheral blood samples from melanoma patients were collected immediately after diagnosis and before the beginning of any treatment. At that moment neither clinical signs of primitive/secondary hematological or immunological alterations (red cell or leukocyte cytopenias as well as leuko (lympho)cytosis) nor detectable infectious or autoimmune diseases were evident.

\section{Power calculation}

We estimated that a sample size of 70 patients and 140 unmatched controls gives a power of $80 \%$ in detecting a

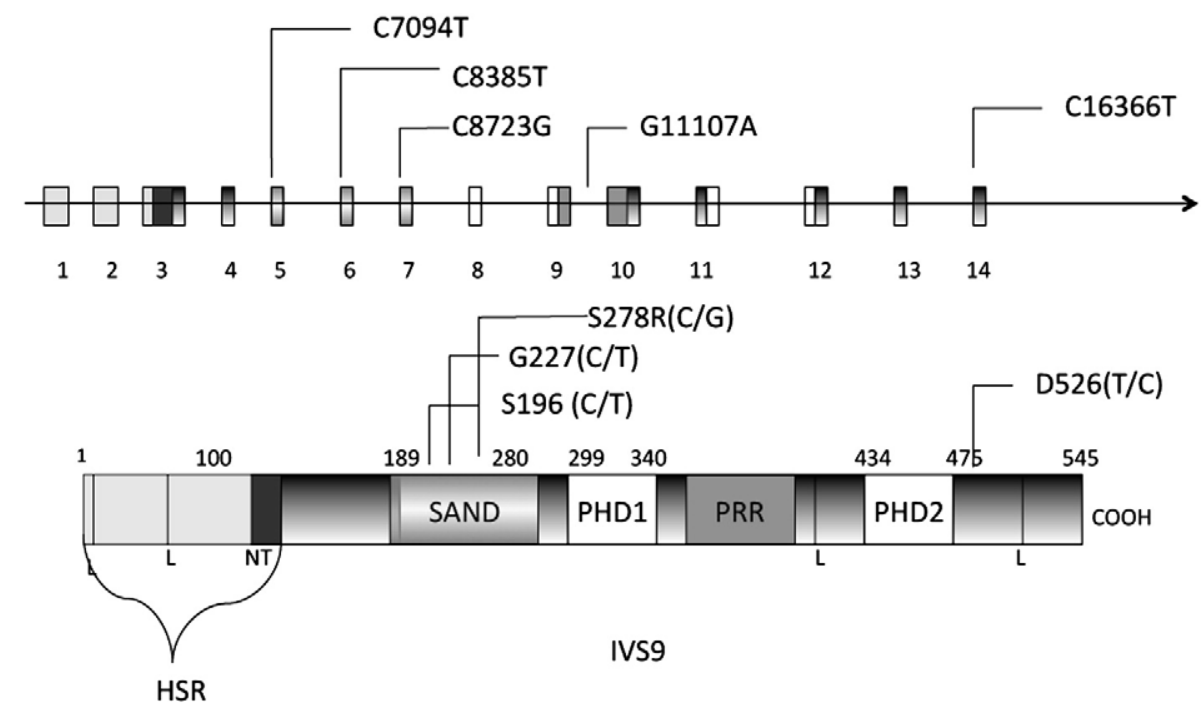

Figure 1 Localization of the five polymorphisms in AIRE gene and AIRE protein domains. 
difference of allelic frequency between cases and controls from $5 \%$ to $15 \%$ at a significance level of $5 \%$.

\section{Genotyping and polymerase chain reaction (PCR) product analysis by sequencing}

DNA was extracted from the peripheral blood lymphocytes through proteinase $\mathrm{K}$ followed by standard salting-out technique. The exons of the AIRE gene and its intronic flanking sequences (GenBank accession no.AB006684) containing the five SNPs were PCR amplified using primers designed to amplify each of the exons. PCR conditions for the AIRE gene were optimized using various magnesium concentrations and annealing temperatures (Table 1). Genomic DNA (50 ng) was run in each PCR reaction. PCR products were then purified from primers by a UltraClean PCR Clean-up Sample Kit (QIAGEN, Hilden, Germany), automatically sequenced using the ABI BigDye Terminator Ready Reaction Mix (Applied Biosystems, Foster City, CA), and analyzed on an ABI 3130XL Genetic Analyzer (Applied Biosystems, Foster City, CA) according to the manufacturer's protocol.

DNA sequence analyses were performed on PCR products amplified using primers specific for the T-cell receptor (TCR) beta chains of the HLA-CW*16-restricted, MAGE-1-specific T-cell clones $81 / 12$ and $26 / 331$ as well as for the HLA-CW*16 intron 3. Used primers were 5'-CTACAATGAGCAGTTCTTCGG-3' forward for the complementary determining region (CDR) 3 of TCR beta chain of anti-MAGE-1 clone 81/12; 5'-CTGGGCACTTGAAGCTTTCT-3' forward for the CDR3 of the TCR beta chain of anti-MAGE-1 clone 26/331; 5'-ACAGCGACCTCGGGTGGGAA-3' reverse for constant region $2\left(C_{\beta 2}\right)$ of the TCR beta chain of anti-MAGE-1 clone $81 / 12$; 5'-CCTGGGTCCACTCGTCATTCTCCG-3' reverse for constant region $1\left(C_{B 1}\right)$ of the TCR beta chain of anti-MAGE-1 clone 26/331; 5'-AGGCCGGGTCTCACACCCTCCA-3' forward for HLA-CW*16 intron 3; 5'-GTTCTCCAGGTATCTGCGGA-3' reverse for HLA-CW*16 intron 3 [26]. The parameters used for amplification were 34 cycles at $94{ }^{\circ} \mathrm{C}$ for $30 \mathrm{~s}, 60{ }^{\circ} \mathrm{C}$ for $30 \mathrm{~s}$ and $72{ }^{\circ} \mathrm{C}$ for $60 \mathrm{~s}$.

\section{Genotyping by real-time PCR}

The polymorphism rs1800520 (C8723G) was investigated using the allelic discrimination assay by real time PCR. An assay detecting melting differences of a probe with the rs1800520 SNP and its corresponding wild-type sequence was utilized in this study. Melting differences were detected using a FRET hybridization quenching assay [27]. PCR was performed using two primers flanking the SNP and two Hybprobes: the 5' located sensor probe, covering the SNP, and the 3' located anchor probe. The sensor probe was designed as a perfect match to the polymorphic allele while the anchor probe was matched to the non-polymorphic sequence immediately 3 ' flanking the SNP region. PCR conditions, primers and Hybprobes specific for the rs1800520 polymorphism were optimized and purchased from TIBmolbiol: forward 5'-CCTTGCAGGGTAGAGGTGAAGCTA-3', reverse 5'-TATTACCATTGCGTATTATCAGG-3', Hybprobe Anchor 5'-GGGTCACTGGGGAGGGCCAGAGG FL-3', Hybprobe Sensor G mut 5'-GCGGGAACCCTGCCCTG PH-3'. The assay was conducted with the LightCycler instrument (Roche Diagnostics) and the software 3 Run version 3.5 was used to analyse probes hybridization and their melting temperature. Ten nanograms of genomic DNA ( $\max 5 \mu \mathrm{l})$ was used per assay. PCR was carried out in three steps: activation $\left(95^{\circ} \mathrm{C}\right.$ for $8 \mathrm{~min}$ ), amplification $\left(95^{\circ} \mathrm{C}\right.$ for $5 \mathrm{~min}, 60^{\circ} \mathrm{C}$ for $5 \mathrm{~min}$, $72{ }^{\circ} \mathrm{C}$ for $\left.15 \mathrm{~min}\right)$, melting $\left(72{ }^{\circ} \mathrm{C}\right.$ for $30 \mathrm{~s}, 95^{\circ} \mathrm{C}$ for $20 \mathrm{~s}$, $40{ }^{\circ} \mathrm{C}$ for $30 \mathrm{~s}, 85^{\circ} \mathrm{C}$ for $1 \mathrm{~min}, 40{ }^{\circ} \mathrm{C}$ for $5 \mathrm{~min}$ ). At least $8 \%$ of all genotyping results including all rare heterozygous genotypes were confirmed by sequencing as described above. Twenty microliters of the PCR reactions was used in these experiments with $5 \mu \mathrm{l}$ of $2 \times$ master mix, $0.4 \mu \mathrm{M}$ of primers, $0.4 \mu \mathrm{M}$ of sensor and anchor probes $3 \mathrm{mM} \mathrm{MgCl} 2$ (Roche Diagnostics).

\section{Quantitative analysis of TCR clonotypes by DNA amplification through real-time PCR}

The analyses were performed on genomic DNA from single patients bearing $0-1$ or 2-3 polymorphic alleles. Twenty nanograms of genomic DNA, $14.4 \mu \mathrm{l}$ of nuclease-free water (Roche Diagnostics), $2 \mu$ l of SYBR Green PCR Master Mix (2X) (Roche Diagnostics), $1.5 \mathrm{mM} \mathrm{MgCl} 2$, and $0.5 \mu \mathrm{M}$ of forward and reverse primers (described in the "Genotyping and PCR product analysis by sequencing" section) specific for antiMAGE-1 clones $81 / 12$ or $26 / 331$ were mixed in a total $20 \mu \mathrm{l}$ volume. Real-time PCRs were performed using the LightCycler instrument (Roche Diagnostics) and SYBR Green fluorescence intensity of DNA samples was analyzed by the software 3 Run (version 3.5) (Roche Diagnostics).

Cycling parameters were as follows: an initial incubation of $10 \mathrm{~min}$ at $95{ }^{\circ} \mathrm{C}$ to activate the DNA polymerase, then 40 cycles of $15 \mathrm{~s}$ at $95^{\circ} \mathrm{C}$ followed by $1 \mathrm{~min}$ at $60{ }^{\circ} \mathrm{C}$. Dissociation curves were generated by incubating the amplicons at $95{ }^{\circ} \mathrm{C}$ for $5 \mathrm{~s}$, reducing the temperature to $60{ }^{\circ} \mathrm{C}$ for $15 \mathrm{~s}$ and increasing the temperature to $95^{\circ} \mathrm{C}$ over a dissociation time of $20 \mathrm{~min}$. Data were analyzed with the LightCycler software to estimate cycle threshold $(\mathrm{Ct})$ values and dissociation curves as well as to confirm the presence of amplicons from TCR beta chain transcripts and their purity. $\mathrm{Ct}$ values are fractional cycle numbers at which fluorescence

Table 1 Primer sequences and PCR conditions.

\begin{tabular}{lllll}
\hline Exon & Forward primer 5'-3' & Revers primer 5'-3' & $\begin{array}{l}\text { Annealing } \\
\text { temperature }\left({ }^{\circ} \mathrm{C}\right)\end{array}$ & $\begin{array}{l}\text { Magnesium } \\
\text { concentration }(\mathrm{mM})\end{array}$ \\
\hline 5 & GATCTGTCACCCGCTGTC & GCGTCTCTCGGCAGCAG & 62 & 1.5 \\
6 & CAGCAGTCACTGACTCCTG & GACCTCACTTTCTCTGAC & 69 & 2 \\
7 & GTGAGGCTCCTCACTTGCGCCTA & GTGGCTTCCCTTCAGGGTCACTGG & 56 & 1.5 \\
9 & CCACCAGGGCTGTGGGAGTTGG & GCTCCTTGAAGAGCCCAC & 65 & 1.5 \\
14 & GGCCTACACGACTGCCAAGGCAG & GTGCACCCGAGTTCAAGTATG & 70 & 1
\end{tabular}


equals the threshold level, which is automatically set to be within the exponential region of the amplification curve where there is a linear relationship between the log of change in fluorescence and cycle number. Dissociation curves are formed plotting rising temperature versus the change in fluorescence/change in temperature [28].

\section{Prediction of RNA secondary structures of AIRE allelic variants}

The potential variations in folding of the RNA secondary structure caused by the synonymous and the non-synonymous mutation of AIRE variants were identified using the Vienna RNA package software [29,31].

\section{Statistical analysis}

Statistically significant differences between genotype as well as T-cell clonotype frequencies were assessed using univariate analyses such as the Fisher's exact test for binary variables and the Student $t$ test for continuous variables. The association between the presence of polymorphic genotypes and independent variables was studied using a multivariate logistic regression analysis. Odds ratio (ORs) and 95\% confidence intervals $(95 \% \mathrm{Cls})$ were calculated and $P$ values lower than 0.05 were considered statistically significant. Analyses were performed using SPSS 13.0 and Graphpad Prism 4 softwares.

\section{Results}

\section{Reduced frequency of rs 1055311 (C8385T), rs1800520 (C8723G) and rs1800522 (T16366C) SNPs in melanoma patients compared with controls}

The frequencies of five SNPs of AIRE gene, consisting in the non-synonymous rs1800520 (C8723G) in exon 7, the silent mutations rs878081 (C7094T) in exon 5, rs1055311 (C8385T) in exon 6, rs1800522 (T16366C) in exon 14 and the intronic SNP rs1800525 (G11107A) in intron 9, were analyzed in both patient and control groups.

Concerning nucleotide positions 8385 in exon 6 (rs1055311), 8723 in exon 7 (rs1800520) and 16366 in exon 14 (rs1800522), the corresponding heterozygous genotypes and the homozygous variant genotypes were $(C / T)$ and $(\mathrm{T} / \mathrm{T}),(\mathrm{C} / \mathrm{G})$ and $(\mathrm{G} / \mathrm{G}),(\mathrm{T} / \mathrm{C})$ and $(\mathrm{C} / \mathrm{C})$, respectively. For each SNP, the heterozygous genotype and the homozygous variant genotype, collectively considered, were less frequent in melanoma patients than in healthy controls (OR 0.5 $95 \%, \mathrm{Cl}, 0.2-0.9 ; P=0.03$ for $\mathrm{rs} 1055311$, OR of $0.3,95 \% \mathrm{Cl}$, $0.1-0.6 ; P=0.004$ for $r s 1800520$, OR of $0.5,95 \% \mathrm{Cl}, 0.3-0.9$; $P=0.04$ for $\mathrm{rs} 1800522$, respectively). No statistically significant differences between patients and controls were detected comparing the frequencies of rs878081 SNP in exon 5 and rs1800525 SNP in intron 9. Detailed results of all the analyses are reported in Table 2.

\section{Stratification of the frequencies of rs 1055311 (C8385T), rs1800520 (C8723G) and rs1800522 (T16366C) SNPs for age, sex and melanoma stage}

In order to verify the dependency of the frequencies of rs1055311 (C8385T), rs1800520 (C8723G) and rs1800522 (T16366C) SNPs on independent variables such as age, sex and melanoma stage, univariate and multivariate analyses were performed on the total population of patients and controls. The frequency of rs1055311 (C8385T) SNP was found significantly increased in males than in females $(0$. R.1.9, C.I. 95\% 1.0-3.3; $P=0.02$ ), while the opposite was observed concerning the rs1800520 (C8723G) SNP (O.R. 0.3, C.I. 95\% 0.1-0.7; $P=0.006)$. No statistically significant differences were detected relatively to rs1800522 (T16366C) SNP, and no significant associations of tested SNPs with either age or melanoma stage were detected (not shown).

Table 2 Comparison of the frequency of rs878081 (C7094T), rs1055311 (C8385T), rs1800520 (C8723G), rs1800525 (G11107A) and rs1800522 (T16366C) SNPs between melanoma patients and controls.

\begin{tabular}{|c|c|c|c|c|c|}
\hline \multirow[b]{2}{*}{ Genotypes } & \multirow[b]{2}{*}{$\begin{array}{l}\text { Percent frequency } \\
\text { in controls/patients }\end{array}$} & \multicolumn{2}{|l|}{ Univariate analysis $^{a}$} & \multicolumn{2}{|l|}{ Multivariate analysis $^{\mathrm{b}}$} \\
\hline & & OR $(95 \% \mathrm{Cl})$ & $P$ & OR $(95 \% \mathrm{Cl})$ & $P$ \\
\hline$\frac{\text { rs878081 variant genotypes }}{(\mathrm{CT}+\mathrm{T})}$ & $40 / 36$ & 1 Reference/0.8 (0.4-1.4) & 0.5 & 1 Reference/0.7 (0.3-1.2) & 0.2 \\
\hline$\frac{\text { rs1055311 variant genotypes }}{(\mathrm{CT}+\mathrm{TT})}$ & $34 / 20$ & 1 Reference/0.5 (0.2-0.9) & 0.03 & 1 Reference/0.4 (0.2-0.9) & 0.02 \\
\hline$\frac{\text { rs1800520 variant genotypes }}{(C G+G G)}$ & $28 / 10$ & 1 Reference/0.3 $(0.1-0.6)$ & 0.004 & 1 Reference/0.3 $(0.1-0.6)$ & 0.004 \\
\hline$\frac{\text { rs } 1800525}{(G A+A A)}$ variant genotypes & $26 / 22$ & 1 Reference/0.7 (0.4-1.5) & 0.4 & 1 Reference/0.7 (0.3-1.4) & 0.3 \\
\hline$\frac{\text { rs1800522 variant genotypes }}{(\mathrm{TC}+\mathrm{CC})}$ & $61 / 47$ & 1 Reference/0.5 (0.3-0.9) & 0.043 & 1 Reference/0.5 (0.3-0.9) & 0.048 \\
\hline
\end{tabular}


Frequencies of different associations of rs1055311 (C8385T), rs1800520 (C8723G) and rs1800522 (T16366C) SNPs in the genotypes of patient and control groups

Here we define as CCT (wild-type) genotype the one that is present in subjects who do not carry any of the three rs1055311, rs1800520 and rs1800522 AIRE gene SNPs. The frequency of CCT genotype was significantly higher in subjects with melanoma $(18.5 \%)$ than in healthy individuals (8.7\%, $P=0.05)$. No statistically significant differences between patients and controls were found when all the other possible genotype combinations were considered (Table 3). Accordingly, when the number of borne polymorphisms per subject was considered, individuals with more than one SNP were significantly more frequent in the group of healthy controls compared with melanoma patient group $(P<0.01)$ (Fig. 2).

\section{Polymorphic AIRE mRNAs structure analysis}

Genetic polymorphisms, through nucleotide substitutions, may generate mRNA molecules showing different secondary structures. Interestingly, variants of mRNA secondary structure have been associated with different efficiencies of gene expression depending on the free energy possessed by the single mRNA molecule [30]. Hence, we decided to analyze the impact on mRNA folding of synonymous and non-synonymous mutations constituting the five AIRE SNPs using a computerized modeling analysis. mRNA endowing allelic variants relative to synonymous mutations, consisting in the rs878081 (C7094T), rs1055311 (C8385T) and rs1800522 (T16366C) polymorphisms, showed equivalent free energy, comparable to that of the wild-type genotype $(\mathrm{dG} \approx-574,7 \mathrm{kcal} / \mathrm{mol})$, thus excluding their capacity of generating AIRE mRNA molecules with secondary structure different from that transcribed on the wild-type genotype.

We also investigated the mRNA folding structure corresponding to the AIRE allelic non-synonymous variants AGCG, present in the wild-type genotype, and AGGG, present in the rs 1800520 (C8723G) SNP, in exon 7. mRNA transcribed on AIRE AGCG allele showed lower free energy $(\mathrm{dG}=-$ $574,7 \mathrm{kcal} / \mathrm{mol}$ ) than the one transcribed on the AGGG allele $(\mathrm{dG}=-562.6 \mathrm{kcal} / \mathrm{mol})$, suggesting that the former likely has greater molecular stability than the latter (Fig. 3). Therefore,

Table 3 Frequencies of AIRE genotype combinations in melanoma patients and controls.

\begin{tabular}{llll}
\hline Genotype combinations & \multicolumn{2}{l}{ Allelic frequency } & $P$ \\
\cline { 2 - 3 } & Patients & Controls & \\
\hline 8385T 8723G 16366C: TGC & 0 & 0.03 & 0.9 \\
8385T 8723G 16366T: TGT & 0 & 0.01 & 0.9 \\
8385T 8723C 16366T: TCT & 0.05 & 0.04 & 0.6 \\
8385C 8723C 16366T: CCT & 0.18 & 0.08 & 0.05 \\
8385T 8723C 16366C: TCC & 0.035 & 0.06 & 0.4 \\
8385C 8723G 16366C: CGC & 0.028 & 0.05 & 0.2 \\
8385C 8723G 16366T: CGT & 0.007 & 0.02 & 0.2 \\
8385C 8723C 16366C: CCC & 0.15 & 0.1 & 0.2 \\
\hline
\end{tabular}

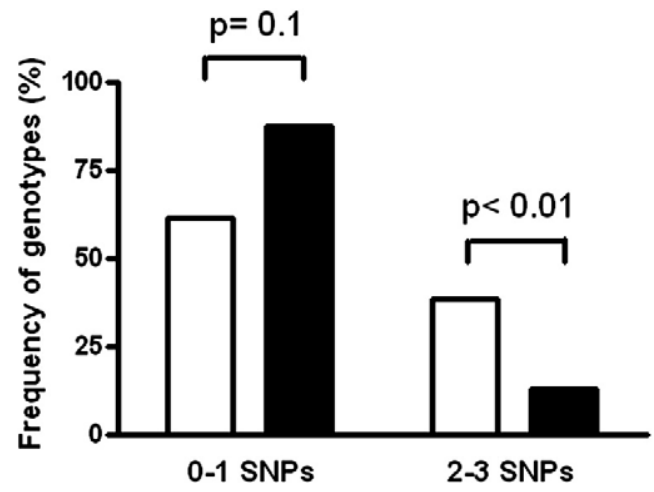

Figure 2 Frequency of genotypes with $0-1$ or 2-3 of the rs1055311, rs1800520 and rs1800522 SNPs in melanoma patients (black bars) and healthy controls (open bars).

mRNA molecules transcribed on AIRE AGCG allele might have a longer half-life and produce more AIRE protein than mRNA molecules transcribed on the variant AGGG genotype.

\section{Quantitation of genomic sequences specific for $81 / 12$ and 26/331 T-cell clonotypes}

Previous studies reported increased frequency of MAAsspecific T-cell clonotypes in melanoma patients with selected HLA haplotypes [26,32,33]. In particular, T-cell clonotypes $81 / 12$ (carrying TCR beta chain regions V5J2S1C2) and 26/331 (carrying TCR beta chain regions V5J1S1C1) were found expanded in HLA-CW*16 positive melanoma patients [26]. In order to verify whether T-cell clonotypes 81/12 and 26/331 were differently represented in the T-cell repertoire of melanoma patients with different AIRE genotypes, we initially screened our series of patients for the presence of the HLA-CW*16 allele. PCR analysis demonstrated that 45 out of 70 patients were positive for the allele of interest. HLA$\mathrm{CW}^{*} 16$ positive patients were subsequently divided in two groups: group A including subjects carrying the wild-type genotype or carrying only one protective SNP; group B including individuals carrying more than one SNP. Genomic DNA from each patient was amplified by RT-PCR until the threshold value. Two ranges of cycle numbers that needed to reach the threshold value were considered for each clonotype: 30-33 and 34-36 for clonotype 81/12, and 26-29 and 30-31 for clonotype 26/331. Considering clonotype 81/ 12 . DNA amplification reached the threshold value in the early (30-33 cycles) range of cycle number in the $91 \%$ of group $B$ patients but only in the $14 \%$ of group A patients $(P=0.001)$. Considering clonotype $26 / 331$, DNA amplification reached the threshold value in the early (26-29 cycles) range of cycle number in the $89 \%$ of group B patients but only in the $20 \%$ of group A patients $(P=0.05)$ (Fig. 4). Hence, these data unveil an overrepresentation of both T-cell clonotypes in patients with 2 or more AIRE SNPs with respect to patients with wild-type genotype or only one SNP.

\section{Discussion}

The results of this study show that (a) AIRE SNPs rs1055311 (C8385T), rs1800520 (C8723G), and rs1800522 (T16366C) are 

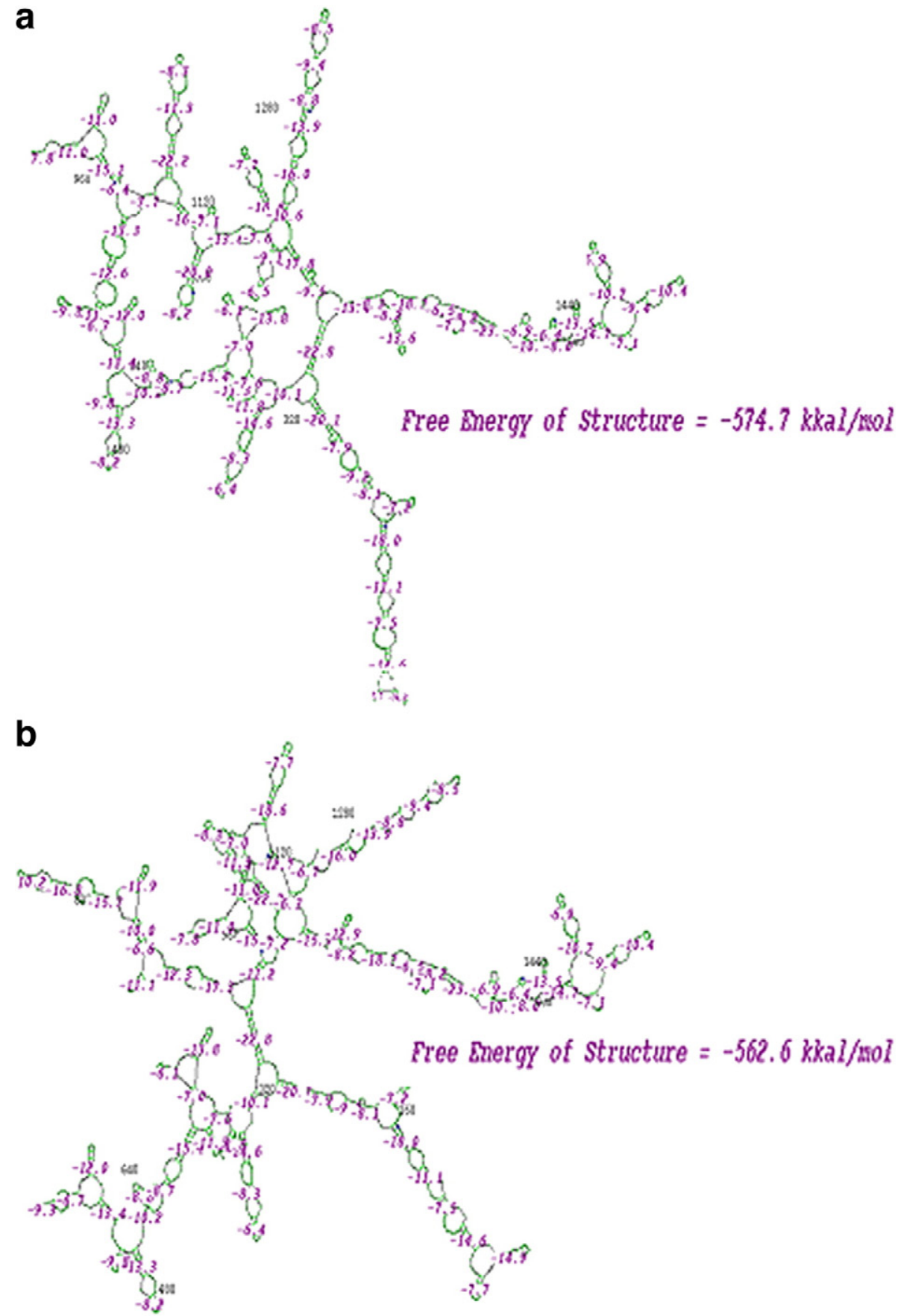

Figure 3 Computerized modeling of AIRE mRNA structures of two haplotype variants in exon7. (a) AGCG allelic variant corresponding to the wild-type genotype; (b) AGGG allelic variant corresponding to the C8723G SNP.

more frequent in the healthy population than in patients with melanoma; (b) the free energy of the mRNA transcribed on AIRE rs1800520 (C8723G) SNP gene sequence is higher than that of the mRNA transcribed on the corresponding wild-type genotype; and (c) two MAGE-1-specific T-cell clonotypes were found more represented in patients with two or more AIRE SNPs than in those with less than two AIRE SNPS.

AIRE induces the transcription of tissue-associated antigens in $\mathrm{mTEC}$, which is important for the negative selection of autologous cytolytic $\mathrm{T}$ lymphocytes $(\mathrm{CTL})$ that recognize autoantigens. Polymorphic AIRE molecules may differently regulate selection of thymocytes potentially able to recognize MAAs once malignant melanoma cells should appear in the organism. On this basis, we decided to analyze SNPs of AIRE gene in order to verify whether peculiar polymorphic genotypes could protect or predispose to the development of melanoma. Among all the AIRE SNPs already identified, five SNPs were selected following two main criteria consisting in their presence at elevated frequency in the European population (rs878081, rs1055311, rs1800520, rs1800525 and rs1800522) [34] and in the already demonstrated association 

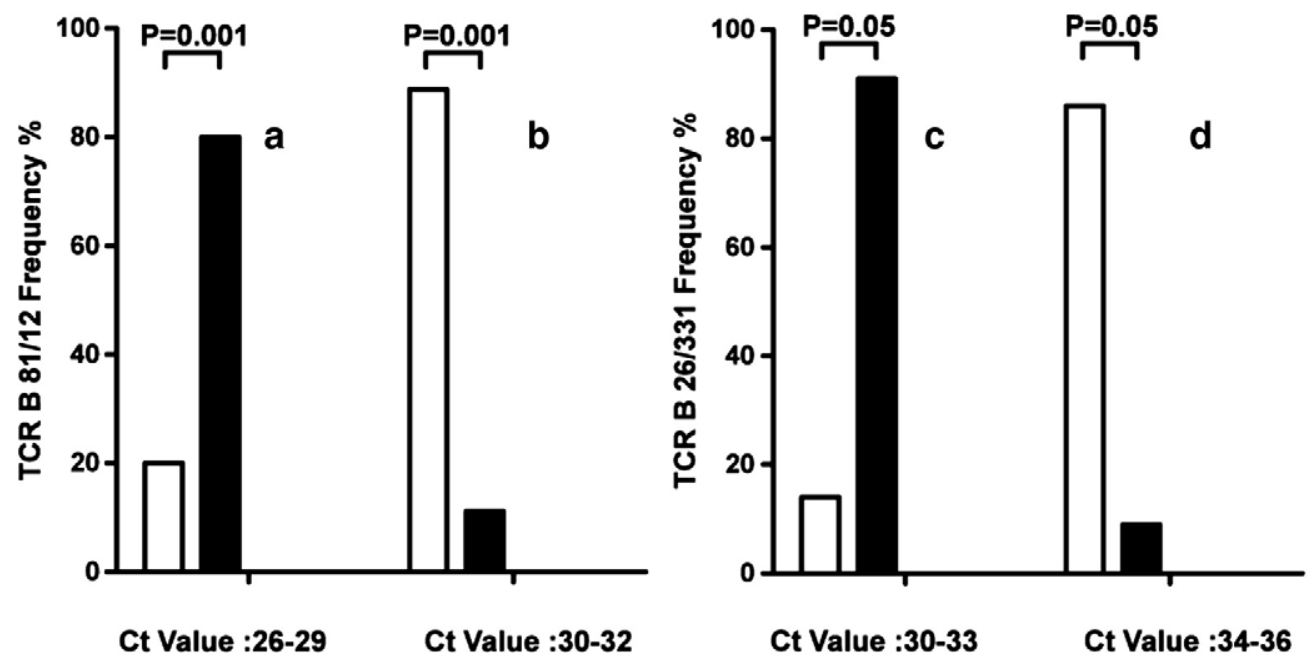

Figure 4 Quantitation of genomic sequences specific for T-cell clonotype 81/12 and 26/331 in HLA-CW*16 positive patients. Early and late ranges of cycle numbers of DNA amplification until threshold value were 30-33 and 34-36 cycles for clonotype 81/12 and 26-29 and 30-33 for clonotype 26/331. Data are expressed as percentages of patients in whom the threshold value was reached in one specific range (early or late) of cycle numbers of DNA amplification. Panels a and b refer to T-cell clonotype 81/12; panels c and d refer to T-cell clonotype 26/331. Panels a and c refer to the early range of cycle number of DNA amplification; panels $b$ and $d$ refer to late ranges of cycle numbers of DNA amplification. Open bars: patients carrying the wild-type genotype or only one protective SNP; black bars: patients carrying more than one SNP.

with human autoimmune diseases (rs1800520 and rs1800525 linked to alopecia aerata and systemic sclerosis associated with autoimmune thyroiditis, respectively) [35,36]. Indeed, we reasoned that an AIRE polymorphic genotype potentially predisposing to an autoimmune disease could also be responsible for a lower level of TRAs transcription in the thymus, including that of tumor-specific antigens, subsequently resulting in a less effective negative selection of autoantigen (and tumor antigen)-specific T lymphocytes and to a potentially lower susceptibility to tumor development. Accordingly, recent studies reported that the expression levels of AIRE and TRAs in mTECs vary considerably from person to person and that mRNA levels of some TRAs are closely correlated to those of AIRE itself [37]. Thus, AIRE genetic polymorphisms cause interindividual variability of AIRE-regulated transcriptional programs in mTECs affecting the levels and fine specificity of ectopic transcripts [38,39]. Hence, opposite differences in tissue-specific antigen expression might result either in escaping of high-avidity antigen-specific $T$ cells (in the case of low intrathymic TRAs expression) or in depleting the T-cell repertoire of clones specific for single (auto)antigens (in the case of elevated intrathymic expression of the corresponding TRA). Remarkably, a direct gene dosage effect between the level of antigen expression, the degree of T-cell deletion/tolerance and the incidence of autoimmunity has been observed in certain experimental models, thus showing the relevance of AIRE-dependent fine regulation of tissue-specific antigen expression [40-44]. Studies performed in T-cell receptor (TCR) transgenic mice have established that the efficiency of negative selection depends on TCR avidity and on the dose-dependent intrathymic self-antigen expression [45-49]. Our data showing the increased representation of specific AIRE SNPs in healthy subjects with respect to melanoma patients suggest that these polymorphisms may be associated with a reduced intrathymic expression of melanoma-associated antigens in individuals bearing the corresponding genotypes, thus conferring them a potential protection against tumor development. Studies are already ongoing in our laboratory aimed at verifying whether AIRE inhibition in mTECs leads to reduced expression of melanomaassociated antigens.

In the attempt of characterizing at the molecular level the mechanisms determining the different functional activity of AIRE allelic variants, we focused our analysis on mRNA folding structure. Various studies in Escherichia coli and in Drosophila have shown that there is a selective translation of the synonymous codons that stabilize the mRNA structure, thus rendering the mRNA molecule more resistant to degradation [50]. Accordingly, codon bias might result from selection in favor of mRNAs with lower folding free energies [51]. Furthermore, additional evidences demonstrate that small stretches of stem-loops in mRNA structure induced by nucleotide substitutions are important for the formation of stable elongation complexes as well as for termination of transcription: therefore this observation confirms that nucleotide substitutions could influence mRNA stability and regulation of gene expression $[52,53]$. Our in silico analysis of the folding of the polymorphic AIRE mRNA structures showed that the genotype corresponding to rs 1800520 SNP leads to the transcription of a mRNA molecule whose secondary structure is likely more unstable than the one of the mRNA coded by the AIRE wild-type genotype, being provided with higher free energy. This observation suggests that, in the case of rs1800520 SNP, AIRE mRNA may have a shorter half life causing reduced AIRE protein production and, thereby, leading to lower melanoma-associated antigen expression in mTECs. Functional studies are ongoing to test this hypothesis.

Other than at the translation level, rs1800520 (C8723G) polymorphism may condition AIRE function at the protein level. In fact, it determines the amino acid change S278R in the SAND domain of the AIRE protein. This domain is responsible for AIRE binding to DNA [54] and for the nuclear localization of 
AIRE $[55,56]$. Therefore, mutations at this domain could destabilize the binding of AIRE to TRAs promoter, thus lowering TRAs expression in thymic epithelial cells.

Whatever the mechanism involved in AIRE modulation of TRAs, the immunological outcome of AIRE function should impact on T-cell repertoire. Considering the case of AIREregulated MAA intrathymic expression, different AIRE genotypes should induce variable representations of MAA-specific T-cell clonotypes. In order to test this hypothesis and to identify an immunological mechanism likely linking expression of specific AIRE genotypes to their protective activity against melanoma development, the frequency of two MAGE-1specific T-cell clonotypes (81/12 and 26/331) was analyzed in patients with less than two AIRE SNPs in comparison with patients carrying two or more AIRE SNPs. The finding that both T-cell clonotypes were more represented in patients with two or more protective AIRE SNPs suggests that these polymorphisms apply a lower selective pressure on MAAs-specific thymocytes than wild-type AIRE gene, leading to a peripheral T-cell repertoire more replenished with MAA-specific T-cell clones. In order to validate this hypothesis, studies will be performed taking into consideration a wider panel of MAAs.

In conclusion, our findings indicate that the variant alleles rs1055311 (C8385T) in exon 6, rs1800520 (C8723G) in exon 7 and rs1800522 (T16366C) in exon 14 exert a significant protective effect against melanoma (Tables 2 and 3). The screening for detecting these polymorphisms in the general population may be useful for identifying subjects with a higher risk of developing the disease being negative for the reported polymorphisms. Moreover, in the context of melanoma patients, the analysis of these polymorphisms could identify the patients who could better benefit from immunotherapy because of presenting the cited polymorphisms and likely possessing a T lymphocyte repertoire covering melanoma-specific antigens. Further studies are warranted to validate these results and to investigate the underlying molecular mechanisms.

\section{Acknowledgments}

This study has been supported by grants from Compagnia di San Paolo, Torino, "Immunoterapia con peptidi telomerasici dei carcinomi della prostata e del rene" and from Fondazione CARIGE "Ricerca sull'immunoterapia del melanoma basata sull'immunizzazione genica e sull'inibizione funzionale dei linfociti soppressori".

We thank TIBmolbiol of Genoa for primers and Hybprobe planning/optimization, and for the excellent technical support.

\section{References}

[1] K. Nagamine, P. Peterson, H.S. Scott, J. Kudoh, S. Minoshima, M. Heino, K.J.E. Krohn, M.D. Lalioti, P.E. Mullis, S.E. Antonarakis, K. Kawasaki, S. Asakawa, F. Ito, N. Shimizu, Positional cloning of the APECED gene, Nat. Genet. 17 (1997) 393-398.

[2] Finnishe German APECED Consortiumm, An autoimmune disease, APECED, caused by mutations in a novel gene featuring two PHDtype zinc-finger domains, Nat. Genet. 17 (1997) 399-403.

[3] P. Bjo" rses, M. Halonen, J.J. Palvimo, M. Kolmer, J. Aaltonen, P. Ellonen, J. Perheentupa, I. Ulmanen, L. Peltonen, Mutations in the
AIRE gene: effects on subcellular location and transactivation function of the autoimmune polyendocrinopathycandidiasis-ectodermal dystrophy protein, Am. J. Hum. Genet. 66 (2000) 378-392.

[4] J. Pitka“nen, V. Doucas, T. Sternsdorf, T. Nakajima, S. Aratani, K. Jensen, H. Will, P. Vähämurto, J. Ollila, M. Vihinen, H.S. Scott, S.E. Antonarakis, J. Kudoht, N. Shimizu, K. Krohn, P. Peterson, The autoimmune regulator protein has transcriptional transactivating properties and interacts with the common coactivator CREB-binding protein, J. Biol. Chem. 275 (2000) 16802-16809.

[5] L. Mittaz, C. Rossier, M. Heino, P. Peterson, K.J. Krohn, A. Gos, M.A. Morris, J. Kudoh, N. Shimizu, S.E. Antonarakis, H.S. Scott, Isolation and characterization of the mouse Aire gene, Biochem. Biophys. Res. Commun. 255 (1999) 483-490.

[6] M.H. Cheng, A.K. Shum, M.S. Anderson, What's new in Aire? Trends Immunol. 28 (2007) 322-327.

[7] M. Halonen, M.P. Huikko, P. Eskelin, L. Peltonen, I. Ulmanen, M. Kolmer, Subcellular location and expression pattern of autoimmune regulator (Aire), the mouse orthologue for human gene defective in autoimmune polyendocrinopathy candidiasis ectodermal dystrophy (APECED), J. Histochem. Cytochem. 49 (2001) 197-208.

[8] D.M. Heery, E. Kalkhoven, S. Hoare, M.G. Parker, A signature motif in transcriptional co-activators mediates binding to nuclear receptors, Nature 387 (1997) 733-736.

[9] D. Uchida, S. Hatakeyama, A. Matsushima, H. Han, S. Ishido, H. Hotta, J. Kudoh, N. Shimizu, V. Doucas, K.I. Nakayama, N. Kuroda, M. Matsumoto, AIRE functions as an E3 ubiquitin ligase, J. Exp. Med. 199 (2004) 167-172.

[10] J. Perheentupa, APS-I/APECED: the clinical disease and therapy, Endocrinol. Metab. Clin. North Am. 31 (2002) 295-320.

[11] P. Bjo"rses, M. Pelto-Huikko, J. Kaukonen, J. Aaltonen, L. Peltonen, I. Ulmanen, Localization of the APECED protein in distinct nuclear structures, Hum. Mol. Genet. 8 (1999) 259-266.

[12] M. Heino, P. Peterson, J. Kudoh, K. Nagamine, A. Lagerstedt, V. Ovod, A. Ranki, I. Rantala, M. Nieminen, J. Tuukkanen, H.S. Scott, S.E. Antonarakis, N. Shimizu, K. Krohn, Autoimmune regulator is expressed in the cells regulating immune tolerance in thymus medulla, Biochem. Biophys. Res. Commun. 257 (1999) 821-825.

[13] M. Heino, P. Peterson, L. Sillanpa, S. Guerin, L. Wu, G. Anderson, H.S. Scott, S.E. Antonarakis, J. Kudoh, N. Shimizu, E.J. Jenkinson, P. Naquet, K.J.E. Krohn, RNA and protein expression of the murine autoimmune regulator gene (Aire) in normal, RelB-deficient and in NOD mouse, Eur. J. Immunol. 30 (2000) 1884-1893.

[14] J.B. Johnnidis, E.S. Venanzi, D.J. Taxman, J.P.-Y. Ting, C.O. Benoist, D.J. Mathis, Chromosomal clustering of genes controlled by the aire transcription factor, PNAS 102 (2005) 7233-7238.

[15] J. Derbinski, A. Schulte, B. Kyewski, L. Klein, Promiscuous gene expression in medullary thymic epithelial cells mirrors the peripheral self, Nat. Immunol. 2 (2001) 1032-1039.

[16] M.S. Anderson, E.S. Venanzi, L. Klein, Z. Chen, S.P. Berzins, S.J. Turley, H. Boehmer, R. Bronson, A. Dierich, C. Benoist, D. Mathis, Projection of an immunological self shadow within the thymus by the AIRE protein, Science 298 (2002) 1395-1401.

[17] L.S. Walker, A.K. Abul-Abbas, The enemy within: keeping selfreactive $T$ cells at bay in the periphery, Nat. Rev. Immunol. 2 (2002) 11-19.

[18] T. Boon, J.C. Cerottini, B.V. Eynde, P. Bruggen, A. Van Pel, Tumor antigens recognized by $T$ lymphocytes, Annu. Rev. Immunol. 12 (1994) 337-365.

[19] C. Öhlén, M. Kalos, L.E. Cheng, A.C. Shur, D.J. Hong, B.D. Carson, T. NC, C.G. Lerner Kokot, B.D. Sather, E.S. Huseby, P.D. Greenberg, CD8 T cell tolerance to a tumor-associated antigen is maintained at the level of expansion rather than effector function, J. Exp. Med. 11 (2002) 1407-1418.

[20] R. Taubert, J. Schwendemann, B. Kyewski, Highly variable expression of tissue-restricted self- antigens in human thymus: 
implications for self-tolerance and autoimmunity, Eur. J. Immunol. 37 (2007) 838-848.

[21] C. Van der Bruggen, C. Traversari, P. Chomez, E. Lurquin, B. De Plaen, A. Van den Eynde, A. Knuth, T. Boon, A gene encoding an antigen recognized by cytolytic $T$ lymphocytes on a human melanoma, Science 254 (1991) 1643-1647.

[22] J. Gotter, B. Kyewski, Regulating self-tolerance by deregulating gene expression, Curr. Opin. Immunol. 16 (2004) 741-745.

[23] B. Kyewski, J. Derbinski, J. Gotter, L. Klein, Promiscuous gene expression and central T-cell tolerance: more than meets the eye, Trends Immunol. 23 (2002) 364-371.

[24] L. Klein, M. Klugmann, K.A. Nave, V.K. Tuohy, B. Kyewski, Shaping of the autoreactive T-cell repertoire by a splice variant of self protein expressed in thymic epithelial cells, Nat. Med. 6 (2000) $56-61$.

[25] J. Gotter, B. Brors, M. Hergenhahn, B. Kyewski, Medullary epithelial cells of the human thymus express a highly diverse selection of tissue-specific genes colocalized in chromosomal clusters, J. Exp. Med. 199 (2004) 155-166.

[26] C. Farina, P. Boel, G. Parmiani, M. Sensi, Conserved TCR usage by HLA-CW*1601-restricted T cell clones recognizing melanoma antigens, Int. Immunol. 8 (1996) 1463-1466.

[27] J.A. Nicklas, E. Blue, A real-time multiplex SNP melting assay to discriminate individuals, J. Forensic Sci. 53 (2008) 1316-1324.

[28] P. Wettstein, M. Strausbauch, T. Therneau, N. Borson, The application of real-time PCR to the analysis of T cell repertoires, Nucleic Acids Res. 36 (21) (2008) e 140.

[29] I.L. Hofacker, RNA secondary structure analysis using the Vienna RNA package, Curr. Protoc. Bioinform. 12 (2004) Unit 12.2.

[30] I.L. Hofacker, Vienna RNA secondary structure server, Nucleic Acids Res. 31 (2003) 3429-3431.

[31] J. Duan, M.S. Wainwright, J.M. Comeron, N. Saitou, A.R. Sanders, J. Gelernter, P.V. Gejman, Synonymous mutations in the human dopamine receptor D2 (DRD2) affect mRNA stability and synthesis of the receptor, Hum. Mol. Genet. 12 (2003) 205-216.

[32] M. Sensi, S. Salvi, C. Castelli, C. Maccalli, A. Mazzocchi, R. Mortarini, G. Nicolini, M. Herlyn, G. Parmiani, A. Anichini, T cell receptor (TCR) structure of autologous melanoma-reactive cytotoxic $\mathrm{T}$ lymphocyte (CTL) clones: tumor-infiltrating lymphocytes overexpress in vivo the TCR beta chain sequence used by an HLA-A2-restricted and melanocyte-lineage-specific CTL clone, J. Exp. Med. 178 (1993) 1231-1246.

[33] J. Shilyansky, M.I. Nishimura, J.R. Yannelli, Y. Kawakami, L.S. Jacknin, P. Charmley, S.A. Rosenberg, T-cell receptor usage by melanoma-specific clonal and highly oligoclonal tumor-infiltrating lymphocyte lines, Proc. Natl. Acad. Sci. U. S. A. 91 (1994) 2829-2833.

[34] S.T. Sherry, M.-H. Ward, M. Kholodov, J. Baker, L. Phan, E.M. Smigielski, K. Sirotkin, dbSNP: the NCBI database of genetic variation, Nucleic Acids Res. 29 (2001) 308-311.

[35] R. Tazi-Ahnini, M.J. Cork, D.J. Gawkrodger, M.P. Birch, D. Wengraf, A.J. McDonagh, A.G. Messenger, Role of the autoimmune regulator (AIRE) gene in alopecia areata: strong association of a potentially functional AIRE polymorphism with alopecia universalis, Tissue Antigens 60 (2002) 489-495.

[36] F. Ferrera, M. Rizzi, B. Sprecacenere, P. Balestra, M. Sessarego, A. DiCarlo, G. Filaci, A. Gabrielli, R. Ravazzolo, F. Indiveri, AIRE gene polymorphisms in systemic sclerosis associated with autoimmune thyroiditis, Clinical. Immunol. 122 (2007) 13-17.

[37] M.S. Anderson, E.S. Venanzi, L. Klein, Z. Chen, S.P. Berzins, S.J. Turley, H. Von boehmer, R. Bronson, A. Dierich, C. Benoist, D. Mathis, Projection of an immunological self shadow within the thymus by the AIRE protein, Science 298 (2002) 1395-1401.

[38] C. Ramsey, S. Hässler, P. Marits, O. Kämpe, C.D. Surh, L. Peltonen, O. Winqvist, Increased antigen presenting cellmediated $\mathrm{T}$ cell activation in mice and patients without the autoimmune regulator, Eur. J. Immunol. 36 (2006) 305-317.
[39] R. Taubert, J. Schwendemann, B. Kyewski, Highly variable expression of tissue-restricted self-antigens in human thymus: Implications for self-tolerance and autoimmunity, Eur. J. Immunol. 37 (2007) 838-848.

[40] J.A. Turunen, M. Wessman, C. Forsblom, R. Kilpikari, M. Parkkonen, N. Pontynen, T. Ilmarinen, I. Ulmanen, L. Peltonen, P.H. Groop, Association analysis of the AIRE and insulin genes in Finnish type 1 diabetic patients, Immunogenetics 58 (2006) 331-338.

[41] A.A. Chentoufi, C. Polychronakos, Insulin expression levels in the thymus modulate insulin-specific autoreactive T-cell tolerance: the mechanism by which the IDDM2 locus may predispose to diabetes, Diabetes 51 (2002) 1383-1390.

[42] K. Miyamoto, S. Miyake, M. Schachner, T. Yamamura, Heterozygous null mutation of myelin P0 protein enhances susceptibility to autoimmune neuritis targeting P0 peptide, Eur. J. Immunol. 33 (2003) 656-665.

[43] K. Thebault-Baumont, D. Dubois-Laforgue, P. Krief, J.P. Briand, P. Halbout, K. Vallon Geoffroy, J. Morin, J. Karine, V. Laloux, A. Lehuen, J.C. Carel, J. Jami, S. Muller, C. Boitard, Acceleration of type 1 diabetes mellitus in proinsulin 2-deficient NOD mice, J. Clin. Invest. 111 (2003) 851-857.

[44] A. Liston, D.H. Gray, S. Lesage, A.L. Fletcher, J. Wilson, K.E. Webster, H.S. Scott, R.L. Boyd, L. Peltonen, C.C. Goodnow, Gene dosage-limiting role of Aire in thymic expression, clonal deletion, and organ-specific autoimmunity, J. Exp. Med. 200 (2004) 1015-1026.

[45] C. Ramsey, A. Bukrinsky, L. Peltonen, Systematic mutagenesis of the functional domains of AIRE reveals their role in intracellular targeting, Hum. Mol. Genet. 11 (2002) 3299-3308.

[46] P.G. Ashton-Rickardt, A. Bandeira, J.R. Delaney, L. Van Kaer, H.P. Pircher, R.M. Zinkernagel, S. Tonegawa, Evidence for a differential avidity model of T cell selection in the thymus, Cell 76 (1994) 651-663.

[47] E. Sebzda, V.A. Wallace, J. Mayer, R.S. Yeung, T.W. Mak, P.S. Ohashi, Positive and negative thymocyte selection induced by different concentrations of a single peptide, Science 263 (1994) $1615-1618$.

[48] E. Robey, B.J. Fowlkes, Selective events in T cell development, Annu. Rev. Immunol. 12 (1994) 675-705.

[49] G.Y. Liu, P.J. Fairchild, R.M. Smith, J.R. Prowle, D. Kioussis, D.C. Wraith, Low avidity recognition of self-antigen by $T$ cells permits escape from central tolerance, Immunity 3 (1995) 407-415.

[50] Y. Chen, D.B. Carlini, J.F. Baines, J. Parsch, J.M. Braverman, S. Tanda, W. Stephan, RNA secondary structure and compensatory evolution, Genes. Genet. Syst. 74 (1999) 271-286.

[51] M. Kashlev, N. Komissarova, Transcription termination: primary intermediates and secondary adducts, J. Biol. Chem. 277 (2002) 14501-14508.

[52] T.C. Reeder, D.K. Hawley, Promoter proximal sequences modulate RNA polymerase II elongation by a novel mechanism, Cell 87 (1996) 767-777.

[53] A. Ujvari, M. Pal, D.S. Luse, RNA polymerase II transcription complexes may become arrested if the nascent RNA is shortened to less than 50 nucleotides, J. Biol. Chem. 277 (2002) 32527-32537.

[54] D.A. Wengraf, A.J.G. McDonagh, T.R.J. Lovewell, Y. Vasilopoulos, S.P. Macdonald-Hull, M.J. Cork, A.G. Messenger, R. Tazi-Ahnini, Genetic analysis of autoimmune regulator haplotypes in alopecia areata, Tissue Antigens 71 (2007) 206-212.

[55] T.J. Gibson, C. Ramu, C. Gemund, R. Aasland, The APECED polyglandular autoimmune syndrome protein, AIRE-1, contains the SAND domain and is probably a transcription factor, Trends Biochem. Sci. 23 (1998) 242-244.

[56] C. Ramsey, A. Bukrinsky, L. Peltonen, Systematic mutagenesis of the functional domains of AIRE reveals their role in intracellular targeting, Hum. Mol. Genet. 11 (2002) 3299-3308. 